Nota de investigación

\title{
Detección de Argyrotaenia sphaleropa Meyrick (Lepidoptera: Tortricidae) en cultivos de Persea americana Mill.
}

Agustina Valverde Rodríguez ${ }^{1 \S}$

Neccy Anel Pérez Velasquez ${ }^{2}$

Miltao Edelio Campos Albornoz ${ }^{1}$

Fernando Jeremías Gonzales Pariona ${ }^{2}$

${ }^{1}$ Facultad de Ciencias Agrarias-Universidad Hermilio Valdizán de Perú. Av. Juan Velasco km 2.1, Pillco Marca, Huánuco, Perú. CP. 10003. (miltao79@ hotmail.com). ${ }^{2}$ Centro de Investigación Olericola Frutícola de Perú-UNHEVAL. Por Carretera 3N km 430.2, Yanac, Huánuco, Perú. CP. 10000. (fgonzales@unheval.edu.pe).

${ }^{\S}$ Autora para correspondencia: avalverde@unheval.edu.pe.

\section{Resumen}

En el Centro de Investigación Frutícola Olerícola de la Facultad de Ciencias Agrarias de la Universidad Nacional Hermilio Valdizán, se sitúa el Banco de Germoplasma de 22 variedades de palto. Durante el periodo marzo-agosto, 2019 se detectó la presencia de la especie Argyrotaenia sphaleropa Meyrick, atacando al cultivo del palto. Se muestrearon frutos describiendo las características del daño. Así también las larvas y pupas encontradas fueron trasladadas al laboratorio para la crianza y su descripción morfológica en base a la clave dicotómica del orden. Se observó que las larvas iniciaban su alimentación con raspaduras irregulares del epicarpio pudiendo afectar hasta el mesocarpo del fruto y a su paso dejaban deyecciones de color blanco, unían las hojas y los frutos entre sí con hilos de seda sirviéndoles de refugio a las larvas para el proceso de pupa y posterior desarrollo del adulto.

Palabras claves: Argyrotaenia sphaleropa, hilos de seda, plaga del palto.

Recibido: octubre de 2021

Aceptado: noviembre de 2021 
En Perú la especie Argyrotaenia sphaleropa Meyrick (1909) (Lepidoptero: Tortricidae) fue detectado causando daños en los cultivos de tara Caesalpinia spinosa (Molina) árbol de origen peruano de importancia industrial (Murga et al., 2015) y en los cítricos (Salazar, 1999; Aliaga, 2014). En Brasil durante la cosecha 2000 y 2001 se registra la especie en los cultivos de durazno (Botton et al., 2003) y en la temporada 1994-1995 se detectó en las plantaciones de cítricos (Citrus sinensis) y recientemente en el cultivo de soja (Neves y da Silva, 2021).

En otros países de Suramérica la ocurrencia de esta plaga se registra en Argentina (Gonsebatt et al., 2018) Bolivia y Uruguay, con ataque a plantas fructíferas (Pinto et al., 2005; Filho et al., 2007), ornamentales y aromáticas (Bentancourt, 1986) en México en el cultivo de palto (Rosas-García y Villegas-Mendoza, 2008).

Es una plaga polífaga frecuentemente con daños en frutales (Rocca y Brown, 2013; Meneguim y Hohmann, 2007) por lo que no se descarta su presencia en el cultivo del palto en Perú. Sin embargo, aún no existe el reporte de la ocurrencia a nivel país, por tanto. El objetivo del presente estudio es reportar la detección de la especie con daños a nivel de frutos en las variedades observadas.

Entre los meses marzo a agosto del año 2019, se realizaron monitoreos y evaluaciones frecuentes de las plagas en el cultivo del palto (Persea americana Mill.) perteneciente al banco de germoplasma de 22 variedades, propiedad del Centro de Investigación Frutícola Olericola de la Facultad de Ciencias Agrarias de la Universidad Nacional Hermilio Valdizán (CIFO) Huánuco-Perú, situado a $1930 \mathrm{msnm}$ en, en donde alberga 22 variedades desde hace aproximadamente 22 años.

Los monitoreos y las observaciones se realizaron en todas las variedades, eligiendo 5 árboles de cada variedad, en las que se realizaron las revisiones visuales alrededor del tercio medio y superior de la planta y procediendo a colectar hojas o frutos dañados por la plaga. Al detectar las pupas en los frutos se colocaron hasta ocho jaulas a base de tull con función de trampeo para permitir que los adultos eclosionen en las condiciones naturales medio ambientales (Figura 1).

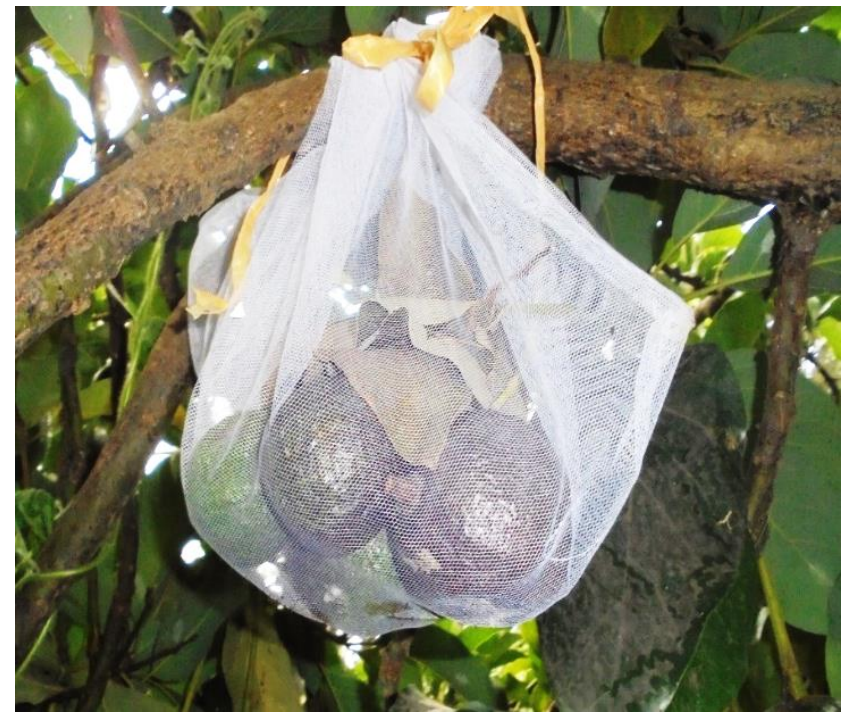

Figura 1. Jaulas a base de tull con frutos dañados conteniendo pupas. 
Se colectaron 47 frutos dañados, 23 larvas de diferentes estadios y 18 pupas Meyrick (1909). Divididos en 16 larvas y 13 pupas de la variedad Hass, cinco larvas y tres pupas de la variedad Naval Verde y dos larvas, dos pupas de la variedad Verónica.

Este material fue trasladado a la zona de crianza entomológica del laboratorio de la Universidad para permitir el desarrollo de adultos, siendo las provisiones de alimentos para las larvas las frutas sanas y para el refugio de las pupas se utilizaron las hojas del palto. Los daños fueron descriptos a través de las observaciones en frutos, hojas y otras estructuras de la planta.

Entre las 22 variedades del palto evaluados, en la variedad Hass se registró el mayor número de frutos dañados (32) que representa $68 \%$ del total de los frutos evaluados, seguida por la variedad Naval Verde con 9 frutos dañados (19\%) y la variedad Verónica con seis frutos dañados (13\%).

Existen diferencias estadísticas significativas entre la cantidad de frutos dañados por variedad $(p \leq$ 0.05 y $p \leq 0.01$ ), siendo Hass la más afectada con un promedio de 6.4 frutos con daño/planta, seguida por la variedad Naval Verde con 1.8 frutos dañados/planta y la variedad Verónica con 1.2 frutos con daño/planta (Cuadro1).

Cuadro 1. Ataque de A. sphaleropa Meyrick en distintas variedades del palto

\begin{tabular}{ccccc}
\hline Variedades & Frutos con daño & EE & 0.05 & 0.01 \\
\hline Hass & $6.4^{* *}$ & 0.28 & $\mathrm{a}$ & $\mathrm{a}$ \\
Naval Verde & 1.8 & 0.28 & $\mathrm{~b}$ & $\mathrm{~b}$ \\
Verónica & 1.2 & 0.28 & $\mathrm{~b}$ & $\mathrm{~b}$ \\
CV $(\%)$ & 19.76 & & & \\
$p$-value & $<0.0001$ & & & \\
\hline
\end{tabular}

Valores en la misma columna seguidos de la misma letra no son estadísticamente diferentes (Duncan, $p \leq 0,05) ;{ }^{* *}=$ valores significativos; $\mathrm{CV}=$ coeficiente de variación; $p$-value $=$ diferencia significativa; $\mathrm{EE}=$ error estándar.

Las larvas de color verde amarillento al principio y verde en los últimos estadios son muy activas fabricando hilos de seda para unir las hojas o frutos entre sí, la unión suele utilizase como escondrijo y a la vez para alimentarse del tejido vegetal parenquimatoso (Figura 3a y 3b). Este comportamiento es parecido al reporte de Juárez (2013) al identificar la especie el cultivo de zarzamora.

Cada larva construye un refugio exclusivo entre las hojas o frutos, inician su alimentación con raspaduras irregulares del epicarpio pudiendo llegar afectar hasta el mesocarpio del fruto y a su paso dejan deyecciones de color blanco, en el mismo fruto dañado y dentro de la tela tejida o en la unión de hojas logra empupar con facilidad (Figuras 2a, 2b y 2c). Similar comportamiento reporta Murga (2015), en las vainas afectadas de Caesalpinia spinosa. Con perforaciones, que varían entre 5 a $20 \mathrm{~mm}$ de diámetro, afectando también a las semillas. 


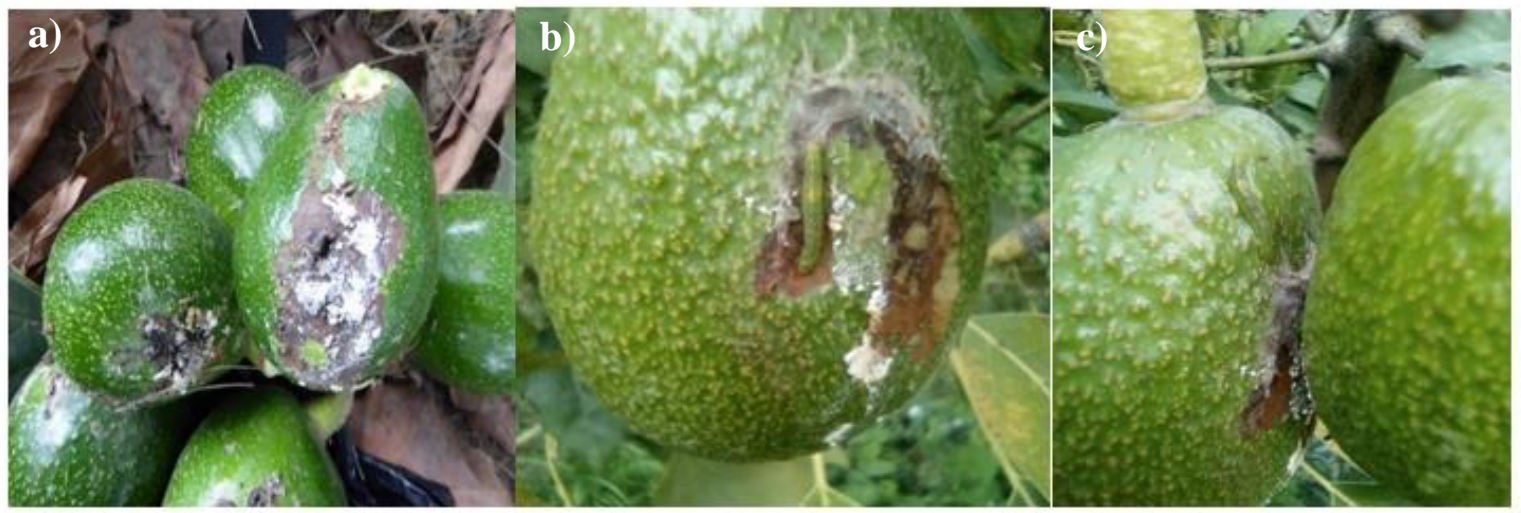

Figura 2. a) daños al epicarpio del fruto; b) deyecciones e hilos de seda; y c) frutos unidos.

Las larvas al ser molestadas tienden a caerse rápidamente apoyado por un hilo de seda que le facilita el salto libre. Las pupas inactivas de color verde al principio y castaño marrón al final completan su ciclo de desarrollo entre los frutos u hojas pegadas entre si (Figura 3c). Se ha observado larvas y pupas ubicadas entre las inserciones de los pedúnculos en la variedad Naval Verde (Figura 3d). Botton et al. (2003) menciona que las larvas raspan la epidermis de los frutos y generalmente se refugian en la inserción del pedúnculo o en las hojas plegadas entre sí o con frutos.

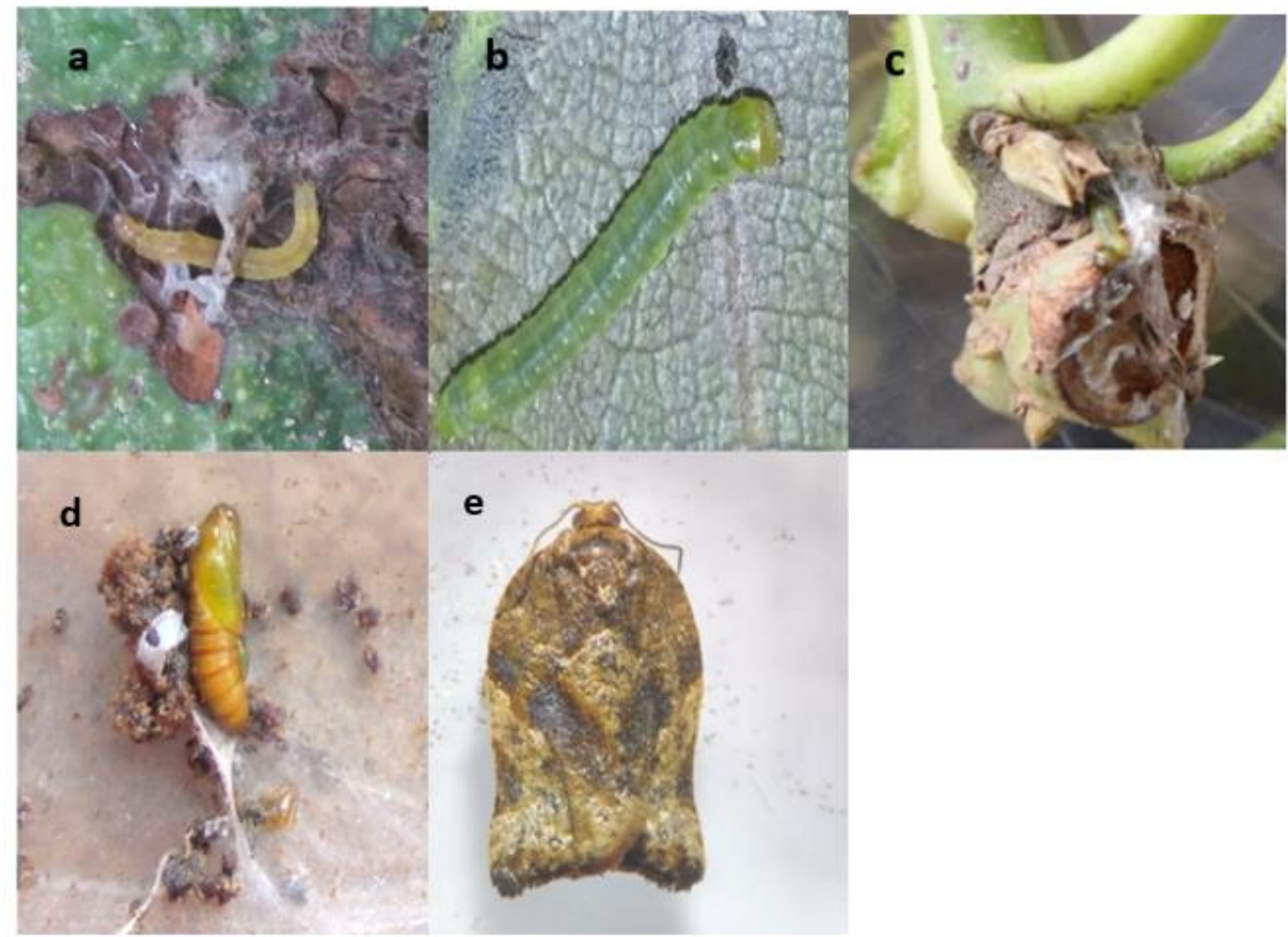

Figura 3. a) larva de color verde amarillento al principio; b) larva de color verde en los últimos estadios; c) pupa; d) larva ubicada en la inserción del pedúnculo; e) adulto. 
Los adultos de hábito nocturno con tamaños de entre $15 \mathrm{~mm}$, con antenas tipo filiforme, provistas de una espiritrompa, tórax y abdomen de color castaño ceniciento, patas de color marrón similar a lo descrito por Bavaresco et al. (2005) son de fácil reconocimiento.

El macho, en la parte dorsal entre las dos alas anteriores forma una figura en forma de ' $\mathrm{V}$ ' de color negro sobre el cual descansa otra figura que se asemeja a un 'rombo', de color claro. Las áreas distantes del ala son de color beige con manchas marrón oscuro en el margen costal-interior y marrón claro entre lados del ángulo apical-anal, margen exterior, las alas posteriores son de color marrón claro. Similar característica es descrita por Neves y da Silva (2021) al realizar el primer registro de A. sphaleropa en cultivo de soja. La hembra adulta es de color marrón claro con manchas irregulares de color negro en las alas anteriores (Figura 3e) y es más grande que el macho.

\section{Conclusiones}

Se confirma la ocurrencia de A. sphaleropa Meyrick (1909) con daños en el cultivo del palto, de preferencia en la variedad Hass.

\section{Literatura citada}

Aliaga, C. J. C. 2014. Fluctuación poblacional de Argyrotaenia sphaleropa (Meyrick) (Lepidoptera: Tortricidae) en mandarina, Huaral-Lima. Universidad Nacional Agraria La Molina, Lima, Perú. 8 p.

Bavaresco, A.; García, M. S.; Botton, M. and Morandi, F. W. J. 2005. Biologia e reprodução de Argyrotaenia sphaleropa. (Lepidoptera: Tortricidae) em folhas de caquizeiro, macieira e videira. Arquivos do Instituto Biológico. 72(3):365-372.

Bentancourt, C. M. 1986. Biología de Argirotaenia Sphaleropa Meyrick. (Lep., Tortricidae) en condiciones de laboratorio cCarlos M. Bentancourt, Iris Beatriz Scatoni. Rev. Brasil. Biol. 46 p.

Botton, M.; Bavaresco, A. and García, M. S. 2003. Ocorrência de Argyrotaenia sphaleropa (Meyrick) (Lepidoptera: Tortricidae) danificando pêssegos na serra gaúcha, Rio Grande do Sul. Neotrop. Entomol. 32(3):503-505.

Filho, W. J. M.; Botton, M.; Grützmacher, A. D. and Nuñez, S. 2007. Flutuação populacional de Argyrotaenia sphaleropa (Lep: Tortricidae) com emprego de feromônio sexual sintético na cultura da videira. Rev. Bras. Frutic. 29(2):213-216.

Gonsebatt, G. F.; Chalup, A. E.; Ruberti, D.; Seta, S.; Leone, A.; Coniglio, R. and Moyano, M. I. 2018. Primer registro de Argyrotaenia tucumana (Lepidoptera: Tortricidae) en montes de duraznero, Prunuspersicae (L.) Batsch, del sur de la provincia de Santa Fe. Rev. de la Sociedad Entomológica Argentina.77(2):28-32.

Juárez Gutiérrez, A. C. 2013. Identificación y biología de un enrollador de hojas de zarzamora: parasitoides asociados a dos tortrícidos (Tesis posgrado). Universidad Michoacana de San Nicolas de Hidalgo, México.

Meneguim, A. M. and Hohmann, C. L. 2007. Argyrotaenia sphaleropa (Meyrick) (Lepidoptera: Tortricidae) in citrus in the State of Paraná, Brazil. Neotrop. Entomol. 36(2):317-319.

Murga, O. H.; Abanto, R. C.; Palomino, R. L. and Polo, V. A. R. 2015. Ocurrencia de Argyrotaenia sphaleropa Meyrick. (Lepidoptero: Tortricidae) en Caesalpinia spinosa (Molina) Kuntze Britton \& Rose. Sci. Agrop. 6(4):329-331. 
Neves, F. W. and Silva, R. A. 2021 Primeiro registro de Argyrotaenia sphaleropa (lepidoptera: tortricidae) na cultura da soja no Estado do Mato Grosso. Braz. J. Animal Environ. Res. 4(1):60-66.

Pinto, A. P.; Santana, J. and Botton, M. 2005. Influência da idade da fêmea na performance reprodutiva e longevidade de Argyrotaenia sphaleropa (Lepidoptera, Tortricidae). Iheringia, Sér. Zool. 95(4):435-440.

Rosas-García, N. M. and Villegas-Mendoza, J. M. 2008. Bionomics of a novel species of Argyrotaenia (Lepidoptera: Tortricidae) presents in Mexican avocado orchards. Acta Zool. Mex. 24(1):129-137.

Salazar Torres, J. 1999. Control de plagas de los cítricos. Perú, Servicio Nacional de Sanidad Agraria. 97 p. 